\title{
Do Aspirin and Other Antiplatelet Drugs Reduce the Mortality in Critically Ill Patients?
}

\author{
Wolfgang Lösche, ${ }^{1}$ Janina Boettel, ${ }^{1}$ Björn Kabisch,, ${ }^{2}$ Johannes Winning, ${ }^{1}$ \\ Ralf A. Claus, ${ }^{1}$ and Michael Bauer ${ }^{1}$ \\ ${ }^{1}$ Center for Sepsis Control and Care, Jena University Hospital, Erlanger Allee 101, 07740 Jena, Germany \\ ${ }^{2}$ Department of Anesthesiology and Intensive Care Medicine, Center for Sepsis Control and Care, \\ University Hospital Jena, Bachstraße 18, Gebäude 12, Eingang A, 07743 Jena, Germany
}

Correspondence should be addressed to Wolfgang Lösche, wolfgang.loesche@med.uni-jena.de

Received 6 June 2011; Accepted 3 October 2011

Academic Editor: Jeanine M. Walenga

Copyright ( $) 2012$ Wolfgang Lösche et al. This is an open access article distributed under the Creative Commons Attribution License, which permits unrestricted use, distribution, and reproduction in any medium, provided the original work is properly cited.

\begin{abstract}
Platelet activation has been implicated in microvascular thrombosis and organ failure in critically ill patients. In the first part the present paper summarises important data on the role of platelets in systemic inflammation and sepsis as well as on the beneficial effects of antiplatelet drugs in animal models of sepsis. In the second part the data of retrospective and prospective observational clinical studies on the effect of aspirin and other antiplatelet drugs in critically ill patients are reviewed. All of these studies have shown that aspirin and other antiplatelet drugs may reduce organ failure and mortality in these patients, even in case of high bleeding risk. From the data reviewed here interventional prospective trials are needed to test whether aspirin and other antiplatelet drugs might offer a novel therapeutic option to prevent organ failure in critically ill patients.
\end{abstract}

\section{Platelets in Systemic Inflammation and Sepsis}

Sepsis and multiple organ failure are leading causes of death in critically ill patients. There is good evidence that blood platelets play an important role in the development of multiple organ failure (MOF) in these patients [1-3]. A decrease in the number of circulating platelets is very often observed when patients develop sepsis and MOF, and thrombocytopenia is a powerful predictor of mortality [46]. During systemic inflammation and infection platelets become activated as indicated by an increase in the number of CD62P-positive platelets and platelet-leukocyte conjugates [7-9]. Different mechanisms may contribute to platelet activation, including imbalance between plasma level of high molecular weight von-Willebrand factor and its cleaving protease, ADAMTS-13 [10-13], and binding of endotoxins to specific receptors at the platelet surface [14-16]. Adhesion of activated platelets within the microcirculation and formation of platelet aggregates contributes to vascular hyperpermeability as well as hypoperfusion [17-20].
However, platelets do not only contribute to the sepsisassociated disturbances of haemostasis, but they also significantly influence inflammatory processes:

(i) release of compounds with well-known pro- or antiinflammatory effects such as cytokines, chemokines, and lipid mediators [21-26],

(ii) activation of the complement system [27, 28],

(iii) release of antibacterial compounds and, together with neutrophils, trapping of bacteria [25, 29-31],

(iv) receptor-mediated adhesion to monocytes, neutrophils, and endothelial cells resulting in changes of cellular functions such as production of cytokines, chemokines, and reactive oxygen species as well as recruitment and immigration of leukocytes at the site of tissue damage $[22,25,26,32]$.

In summary, platelets may contribute to the development of MOF by disturbing blood flow as well as by modulating 
the systemic inflammation. Thus the question arises whether antiplatelet drugs may have a benefit on the outcome in critically ill patients, that is, in patients with systemic inflammation, severe infections, or sepsis.

\section{Antiplatelet Drugs}

Antiplatelet drugs are widely used in patients with cardiovascular disease for the secondary prevention of atherothrombotic events [34-36]. The mostly used drug is aspirin which is an irreversible inhibitor of cyclooxygenase. In platelets aspirin inhibits the formation of thromboxane $A_{2}$ which is a potent platelet activator $[37,38]$. Since aspirin also affects the cyclooxygenase in gastric mucosa which can lead to serious bleeding, it is used as an inhibitor of platelet function for the prevention in patients with risk for atherothrombosis in rather low dosage, that is, $\leq 325 \mathrm{mg} /$ day, and in many patients at dosage lower than $160 \mathrm{mg} /$ day [35, 36, 39-42].

Clopidogrel and the more recently developed drugs prasugrel and ticagrelor are rather specific inhibitors of platelet function. These drugs or their metabolic products interact with the platelet ADP receptor P2Y12, and they are used in combination or instead of aspirin [43-46]. Another group of antiplatelet agents are inhibitors of the glycoprotein IIb/IIIa complex on the platelet surface. These agents block the binding of fibrinogen to the receptor which is essential for platelet aggregation [47-49].

\section{Anti-Inflammatory Effects of Antiplatelet Drugs in Patients with Cardiovascular Diseases}

Many studies have shown that aspirin and clopidogrel not only reduce the risk of atherothrombotic events, but also reduce markers of systemic inflammation including Creactive protein, soluble $\mathrm{CD} 62 \mathrm{P}$ and $\mathrm{CD} 54$, pro-inflammatory cytokines, and platelet-leukocyte conjugates in these patients [50-54]. It is assumed that the anti-inflammatory effects of antiplatelet drugs are mediated by an inhibition of platelet activation [53].

\section{Animal Studies on the Action of Antiplatelet Drugs in Systemic Inflammation and Sepsis}

In the late seventies and in the eighties of the past century some studies on the beneficial effect of inhibitors of prostaglandin and thromboxane synthesis, including aspirin, on the survival in animal models of sepsis were reported [5557]. It was shown that aspirin reduced platelet accumulation in the lung in a mouse model of endotoxinaemia $[58,59]$. Other studies investigated the effects of glycoprotein IIb/IIIa inhibitors. Using monoclonal antibodies, a reduction of thrombotic microangiopathy and ischemic tissue injury in various animal models of endotoxinaemia or sepsis could be shown [60-62]. More recently, the effects of the ADP receptor antagonist clopidogrel in endotoxinaemia were tested. Evangelista et al. [63, 64] reported an inhibition of platelet-dependent leukocyte activation as well as an inhibition of the production of proinflammatory cytokines in mice after endotoxin administration. Our group could recently show that in a similar mouse model clopidogrel prevented endotoxin-induced thrombocytopenia, reduced fibrin deposition in lung tissue, and inhibited the upregulation of some genes, known to be involved in inflammation, in peripheral blood cells [65]. In a mouse model of polymicrobial sepsis Seidel et al. [66] demonstrated that clopidogrel reduced cell damage and liver dysfunction as indicated by reducing the sepsis-mediated increase in serum lactate dehydrogenase activity and serum bilirubin concentration. Using a rat model of endotoxin-induced systemic inflammation Hagiwara et al. [67] reported that clopidogrel attenuated the increase in serum levels of proinflammatory cytokines (TNF $\alpha$, IL- 6 and HMBG1) and the tissue injury in liver and lung. A benefit of clopidogrel was also shown in a rat model of chronic kidney disease [68].

\section{Benefit of Aspirin and Other Antiplatelet Drugs in Critically Ill Patients}

Based on the evidence that platelets play an important role in the development of organ failure in critically ill patients we performed three retrospective clinical studies. As critically ill patients are often elderly people we assumed that some of them might be on antiplatelet drugs for the prevention of thromboembolic events due to cardiovascular diseases. Indeed, $20-25 \%$ of the patients who were included in the analysis had a preexisting medication with aspirin or/and clopidogrel [33, 65]. Even if the administration of aspirin and/or clopidogrel was discontinued during the stay in hospital, inhibition of platelet function should persist for about one week $[69,70]$.

\subsection{Patients Admitted to Hospital with Community Acquired} Pneumonia. In a first study we analysed data from patients who were admitted to the hospital for community acquired pneumonia (CAP). Since statins are discussed to improve the outcome in critically ill patients [71-76], patients with prehospital use of statins were excluded from the study. Two hundred twenty-four patients were enrolled and 38 of them had a preexisting medication with aspirin and 8 were on clopidogrel or ticlopidin for at least 6 month prior to admission to hospital [65]. As endpoints of the study we used the length of stay in the hospital, and the admission to the intensive care unit (ICU) as an indicator of organ failure. Despite the fact that patients on antiplatelet drugs were about 12 years older when compared to those without such preexisting medication, they were less frequently admitted to ICU $(9.1 \%$ versus $26.3 \%)$. This difference was more pronounced when age-matched subgroups were compared: $24.4 \%$ of patients without and only $5.0 \%$ of patients with antiplatelet drugs required ICU treatment. In the agematched subgroups we observed also a significant shorter stay in hospital for the patients on antiplatelet drugs (13.9 \pm 6.2 versus $18.2 \pm 10.2$ days). The beneficial effect of the preexisting medication with antiplatelet drugs was also obvious when stepwise logistic regression analysis was used 


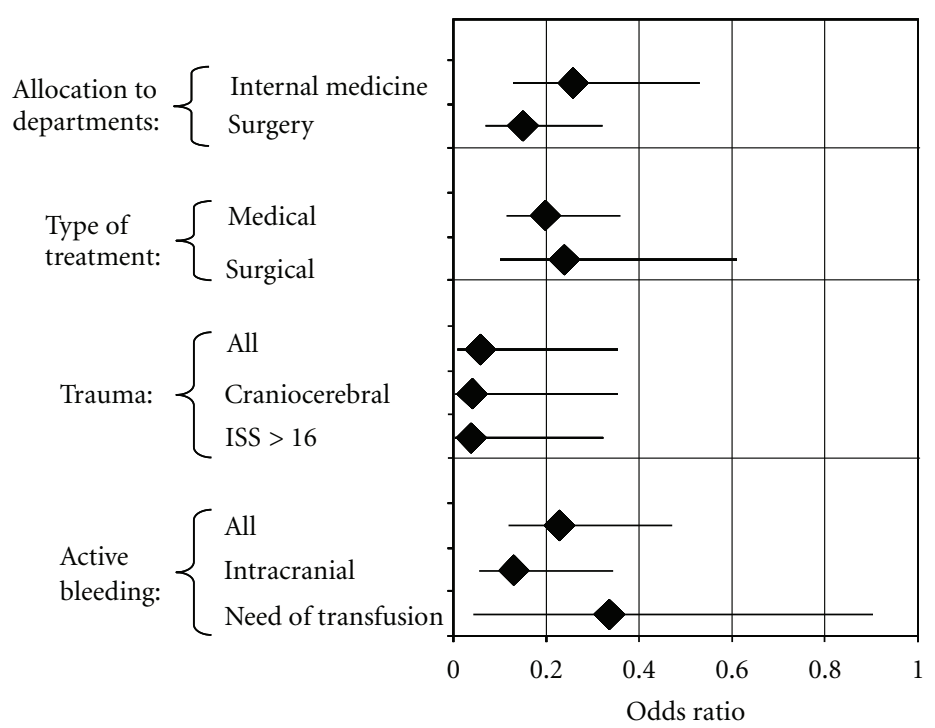

FIGURE 1: The figure summarises the effect of antiplatelet drugs (aspirin or/and clopidogrel) in patients admitted to an ICU as reported by Winning et al. [33]. Odds ratios for ICU mortality with 95\% confidence intervals were calculated by stepwise logistic regression with age, gender, APACHE II score, and preexisting medication with antiplatelet drugs as independent variables.

to calculate the odds ratio for the need of ICU treatment. The following variables were included as independent variables: SOFA (sepsis-related organ failure assessment) score, plasma level of C-reactive protein, platelet and leukocyte counts (all measured at day of admission), age, gender, and the preexisting medication with antiplatelet drugs. Odds ratio of 0.32 (95\% confidence interval: $0.10-1.00)$ for all patients and $0.19(0.04-0.87)$ for the age-matched subgroup were obtained, indicating a marked reduction in organ failure by antiplatelet drugs [65].

5.2. Patients Admitted to an Intensive Care Unit. In a second study we analysed the data from 615 patients who were admitted to the intensive care unit (ICU) within 24 hours after arrival in hospital. From these patients $21 \%$ had a preexisting medication with aspirin $(\leq 160 \mathrm{mg} / \mathrm{d})$, and $4 \%$ were on clopidogrel or a combination of aspirin and clopidogrel. Patients on statins were excluded [33]. Patients were allocated to internal medicine as well as various surgery departments (general surgery, trauma surgery, neurosurgery, and gynaecology). Patients with and without antiplatelet drugs did not only differ in age (median: 72 versus 56 years), but also in the severity of their illness as measured by the APACHE (Acute Physiology and Chronic Health Evaluation) II score at the day of ICU admission (25 versus 19). Despite these marked differences in age and APACHE II score which are established risk factors of MOF, there was no difference in ICU mortality as clinical end point of the study. Stepwise logistic regression with APACHE II score, age, gender, and use of antiplatelet drugs indicated that in addition to age and APACHE II score the use of antiplatelet drugs had a highly significant impact on mortality. The calculated odds ratios amounted for age 1.04 (1.03-1.06), for APACHE II 1.16 (1.12-1.19), and for antiplatelet drugs $0.19(0.12-0.33)$. That means that the prehospital use of antiplatelet drugs would reduce mortality by a factor of about 5 [33].

Figure 1 illustrates the effects of antiplatelet drugs on subgroups of patients [33]. Surprisingly, in patients who were allocated to surgical departments, the preexisting medication with antiplatelet drugs was associated with a slightly better outcome when compared to those patients allocated to internal medicine department. In neurosurgery patients (46 with and 196 without antiplatelet drugs) an odds ratio for mortality of $0.12(0.04-0.30)$ was calculated. In contrast, patients allocated to trauma surgery did not show any benefit from antiplatelet drugs (odds ratio $=0.92(0.06-$ 13.6)) [33]. However, in the subgroup of trauma patients (22 with and 159 without antiplatelet drugs) antiplatelet drugs seemed to exert an enormous benefit as an odds ratio as low as $0.06(0.01-0.35)$ was calculated. This was true for patients with multiple trauma (injury severity score $>16$ ) as well as for patients with craniocerebral trauma (Figure 1). Even patients with active bleeding including those who needed transfusion or presented intracranial bleeding seemed to profit from the preexisting medication with antiplatelet drugs. Finally there was no marked difference in the beneficial effect of antiplatelet drugs between patients who received medical treatment and those with surgical treatment (Figure 1). Thus bleeding and/or high risk for bleeding seemed not to abolish or reverse the calculated benefit of antiplatelet drugs in the critically ill patients. However, one should consider the data obtained by the stepwise logistic regression analysis in some of the subgroups of patients with some caution as (i) the numbers of patients were sometimes rather low and (ii) patients without antiplatelet drugs were much younger (up to 30 years) when compared to those with such medication. Therefore we reevaluated the subgroups in a cohort of APACHE II score and age-matched patients using $2 \times 2$ table analysis [33]. Under this condition the beneficial effect of 
TABle 1: Age, gender, APACHE II score, and mortality in a subgroup of patients with and without an exclusive prehospital aspirin medication. The data were taken from Winning et al. [33].

\begin{tabular}{lccc}
\hline & Control & ASA & Significance \\
\hline $\begin{array}{l}\text { Number } \\
\text { Age (years) }\end{array}$ & 561 & 129 & \\
$\begin{array}{l}\text { Male/female } \\
(\%)\end{array}$ & $56.8 / 43.2$ & $57.4 / 42.6$ & n.s. \\
$\begin{array}{l}\text { APACHE II } \\
\begin{array}{l}\text { Mortality } \\
(\%)^{*}\end{array}\end{array}$ & $19.4 \pm 8.5$ & $26.1 \pm 9.3$ & $P<0.00001$ \\
\hline
\end{tabular}

Data are given as mean \pm standard deviation (sd), total numbers or \%. Significances were calculated either by $t$-test for unpaired samples or by $2 \times$ 2 table analysis. n.s. $=$ not significant. ${ }^{*}$ ICU mortality.

TABLE 2: Effect of aspirin on outcome of critically ill patients characterised in Table 1 . Odds ratios for ICU mortality were calculated using data from our recently published study [33]. The model of stepwise logistic regression included age, gender, APACHE II score, and preexisting medication with aspirin as independent variables.

\begin{tabular}{lc}
\hline Variable & Odds ratio $(95 \%$ CI $)$ \\
\hline Age & $1.04(1.03-1.06)$ \\
APACHE II score & $1.16(1.13-1.20)$ \\
Aspirin & $0.20(0.12-0.35)$ \\
\hline
\end{tabular}

antiplatelet drugs in patients with active bleeding or a high bleeding risk was no longer significant, but the calculated odds ratios for mortality were in most of the subgroups in a range of 0.42 to 0.88 . There were only two exceptions: in patients allocated to the trauma surgery department an odds ratio of $3.67(0.38-42.2)$ was calculated. In contrast, a significant benefit on outcome was still obtained for neurosurgery patients (odds ratio $=0.32(0.12-0.84)$ ) [33] .

For the present paper we have reevaluated our previously reported data [33] summarised above. We analysed the data of those patients who had a preexisting medication with only low-dose aspirin, and we excluded those patients who had clopidogrel or a combination of aspirin and clopidogrel. As for the entire group of patients with antiplatelet drugs we also found for the "only aspirin" subgroup large differences in age and APACHE II score when compared to patients without antiplatelet drugs. And again, there was no difference in mortality (Table 1). However using stepwise logistic regression analysis with mortality as dependent variable and age, APACHE II score, gender, and preexisting aspirin medication, we found that aspirin reduced the mortality by about $80 \%$ as indicated by an odds ratio of 0.20 (Table 2). Thus the calculated benefit of aspirin was in the same range as calculated for the entire group of patients with aspirin and/or clopidogrel as shown in Figure 1 and previously reported [33].

5.3. ICU Patients Presenting Severe Sepsis or Septic Shock. In a third, not yet published study, we analysed the clinical records of 834 patients who were admitted to ICU with severe
TABLE 3: Age, APACHE II score, and mortality in patients admitted to ICU with severe sepsis or septic shock and with or without aspirin medication during ICU stay.

\begin{tabular}{lccl}
\hline \multirow{2}{*}{ Variable } & \multicolumn{2}{c}{ Aspirin during ICU stay } & \multirow{2}{*}{ Significance } \\
& No & Yes & \\
\hline Number & 647 & 187 & \\
Age (years) & $63.4 \pm 14.0$ & $67.9 \pm 12.9$ & $P<0.0001$ \\
APACHE II & $22.6 \pm 9.2$ & $24.1 \pm 8.3$ & $P<0.05$ \\
Mortality (\%) & 33.8 & 23.5 & $P<0.01$ \\
\hline
\end{tabular}

TABLE 4: Effect of ICU aspirin medication on outcome (odds ratio of mortality*) of patients with severe sepsis or septic shock. The model of stepwise logistic regression included age, APACHE II score and ICU medication with aspirin as independent variables. ${ }^{*} \mathrm{ICU}$ mortality.

\begin{tabular}{lc}
\hline Variable & Odds ratio $(95 \% \mathrm{CI})$ \\
\hline APACHE II score & $1.05(1.03-1.07)$ \\
Aspirin & $0.55(0.38-0.81)$ \\
\hline
\end{tabular}

sepsis or septic shock. About $20 \%$ of these patients received low-dose aspirin (Table 3). Exclusion criteria were the administration of other antiplatelet drugs (i.e., clopidogrel) or nonsteroidal anti-inflammatory drugs such as ibuprofen, diclofenac or indomethacin. As shown in Table 3, patients on aspirin were about 4.5 years older and presented a slightly higher APACHE II score at the day of ICU admission. Despite the differences in these both risk factors, ICU mortality was about one third lower in patients receiving aspirin when compared to those without such medication (Table 3 ).

When calculating the odds ratio for mortality by stepwise logistic regression with age, APACHE II score, and aspirin medication during ICU stay, we found a reduction in ICU mortality by aspirin of about $45 \%$ (Table 4 ).

\subsection{Patients at Risk for Acute Lung Injury/Acute Respiratory} Distress Syndrome. In June 2011 O'Neal et al. [73] published the data of a prospective study on the effects of the prehospital use of statins on the prevalence of severe sepsis and acute lung injury/acute respiratory distress syndrome (ALI/ARDS) in critically ill patients. The authors included 575 patients admitted to surgical or medical ICU. Exclusion criteria were admission to trauma or cardiovascular ICU, primary cardiac diagnoses, and age $<40$ years. From these patients 26\% were on prehospital statins. Logistic regression analysis with age, gender, tobacco use, race, APACHE II score, statin use, and aspirin use indicated that patients on statin but not those on aspirin were less likely to have or to develop severe sepsis (odds ratio $0.62,95 \%$ confidence interval 0.40-0.96) or ALI/ARDS (odds ratio 0.60, 95\% confidence interval 0.36-0.99) during the first four days after ICU admission. Interestingly, the benefit of prehospital statins may be potentiated by prehospital aspirin. Patients with both prehospital statins and aspirin had the lowest rate 
of severe sepsis or ALI/ARDS when compared to those with statins alone or those without statins [73].

The association of prehospital aspirin therapy and ALI/ARDS was also investigated by Erlich et al. [77] and published in February 2011. The authors evaluated medical records of 161 patients with at least one major risk factor for ALI/ARDS but who did not meet criteria for ALI/ARDS at time of hospitalisation. Seventy-nine (49\%) of the patients were on aspirin at hospital admission and $33(21 \%)$ developed ALI/ARDS. Aspirin therapy was associated with a significantly lower rate of ALI/ARDS when compared to patients without aspirin (17.7\% versus $28.0 \%$; odds ratio $0.37,95 \%$ confidence interval $0.16-0.84)$. The authors reported that the benefit of aspirin therapy remained significant after adjusting for various confounding variables [77]. A few months later the same group reported the results of a large multicenter international observational study on the association of prehospital aspirin therapy and ALI/ARDS [78]. Inclusion criteria were again at least on risk factor of ALI (aspiration, pneumonia, sepsis, shock, pancreatitis, high-risk trauma, or high risk surgery) and age $>18$ years. Patients with elective surgery were excluded. A total of 3855 patients were enrolled in the study. Twenty-five \% of them were receiving aspirin at the time of hospitalisation and 6.2\% developed ALI/ARDS. Patients with aspirin were significantly older (median and interquartile ranges: 70 (5981) versus 51 (38-66) years) and had higher APACHE II scores $(12(8-16)$ versus $9(5-14))$. Univariate analysis indicated a reduced incidence of ALI/ARDS in patients with aspirin (odds ratio 0.65, 95\% confidence interval 0.46-0.90). However this association was attenuated after adjusting for the propensity to receive aspirin therapy. An odds ratio (Cochran-Mantel-Haenszel pooled odds ratio) of 0.70 (0.481.03) was calculated in a stratified analysis based on deciles of the American Society of Anesthesiologists propensity scores [78].

\section{Discussion}

Animal studies and observational clinical studies reviewed here have provided some evidence that antiplatelet drugs may reduce organ failure and mortality in critically ill patients. In the clinical studies mostly or exclusively aspirin was used as the antiplatelet drug, whereas clopidogrel or GPIIb/IIIa inhibitors as rather specific antiplatelet drugs were predominantly used in animal models [60-68]. The benefit of clopidogrel and GPIIb/IIIa inhibitors in animal models and the benefit of low-dose aspirin in the observational clinical studies may indicate that the benefit of aspirin is indeed mediated by its effect on platelets. However, one cannot exclude the possibility that the benefit of antiplatelet drugs, including aspirin, is at least partially due to the underlying atherosclerotic vascular disease. It is well accepted that atherosclerosis is based on a chronic low-grade systemic inflammation as indicated by moderately increased levels of markers of inflammation, that is, cytokines, C-reactive protein, or fibrinogen [79-82]. It would be interesting to test the hypothesis that patients with chronic low-grade systemic inflammation have a decreased prevalence of severe sepsis and organ failure.

The use of antiplatelet drugs in critically ill patients seems not to be associated with unfavourable bleeding. A benefit of antiplatelet drugs was also evident in patients with an increased bleeding risk such as neurosurgery patients and not necessarily associated with high blood loss or worse neurological outcome [33]. This observation is in line with the recommendation of perioperative continuation of antiplatelet therapy in patients with high risk of cardio- and cerebrovascular events [83-86].

\section{Conclusion}

The data reviewed in the present paper may indicate that low-dose aspirin, as it is used in patients with cardiovascular, cerebrovascular, or peripheral vascular diseases, might offer a novel therapeutic option to prevent organ failure. This hypothesis warrants testing in prospective interventional trials.

\section{References}

[1] M. Levi, "Platelets in sepsis," Hematology, vol. 10, supplement 1, pp. 129-131, 2005.

[2] J. L. Vincent, A. Yagushi, and O. Pradier, "Platelet function in sepsis," Critical Care Medicine, vol. 30, supplement 5, pp. S313-S317, 2002.

[3] J. N. Katz, K. P. Kolappa, and R. C. Becker, "Beyond thrombosis: the versatile platelet in critical illness," Chest, vol. 139, no. 3, pp. 658-668, 2011.

[4] S. Akca, P. Haji-Michael, A. De Mendonça, P. Suter, M. Levi, and J. L. Vincent, "Time course of platelet counts in critically ill patients," Critical Care Medicine, vol. 30, no. 4, pp. 753-756, 2002.

[5] D. Moreau, J. F. Timsit, A. Vesin et al., "Platelet count decline: an early prognostic marker in critically III patients with prolonged ICU stays," Chest, vol. 131, no. 6, pp. 1735-1741, 2007.

[6] D. M. Vandijck, S. I. Blot, J. J. De Waele, E. A. Hoste, K. H. Vandewoude, and J. M. Decruyenaere, "Thrombocytopenia and outcome in critically ill patients with bloodstream infection," Heart and Lung, vol. 39, no. 1, pp. 21-26, 2010.

[7] M. Gawaz, S. Fateh-Moghadam, G. Pilz, H. J. Gurland, and K. Werdan, "Platelet activation and interaction with leucocytes in patients with sepsis or multiple organ failure," European Journal of Clinical Investigation, vol. 25, no. 11, pp. 843-851, 1995.

[8] H. Ogura, T. Kawasaki, H. Tanaka et al., "Activated platelets enhance microparticle formation and platelet-leukocyte interaction in severe trauma and sepsis," Journal of Trauma, vol. 50, no. 5, pp. 801-809, 2001.

[9] S. Russwurm, J. Vickers, A. Meier-Hellmann et al., "Platelet and leukocyte activation correlate with the severity of septic organ dysfunction," Shock, vol. 17, no. 4, pp. 263-268, 2002.

[10] C. L. Bockmeyer, R. A. Claus, U. Budde et al., "Inflammationassociated ADAMTS13 deficiency promotes formation of ultra-large von Willebrand factor," Haematologica, vol. 93, no. 1, pp. 137-140, 2008. 
[11] R. A. Claus, C. L. Bockmeyer, M. Sossdorf, and W. Lösche, "The balance between von-Willebrand factor and its cleaving protease ADAMTS13: biomarker in systemic inflammation and development of organ failure?" Current Molecular Medicine, vol. 10, no. 2, pp. 236-248, 2010.

[12] J. A. K. Hovinga, S. Zeerleder, P. Kessler et al., "ADAMTS-13, von Willebrand factor and related parameters in severe sepsis and septic shock," Journal of Thrombosis and Haemostasis, vol. 5, no. 11, pp. 2284-2290, 2007.

[13] T. N. Bongers, M. Emonts, M. P. M. De Maat et al., "Reduced ADAMTS13 in children with severe meningococcal sepsis is associated with severity and outcome," Thrombosis and Haemostasis, vol. 103, no. 6, pp. 1181-1187, 2010.

[14] G. Andonegui, S. M. Kerfoot, K. McNagny, K. V. J. Ebbert, K. D. Patel, and P. Kubes, "Platelets express functional Toll-like receptor-4," Blood, vol. 106, no. 7, pp. 2417-2423, 2005.

[15] G. Zhang, J. Han, E. J. Welch et al., "Lipopolysaccharide stimulates platelet secretion and potentiates platelet aggregation via TLR4/MyD88 and the cGMP-dependent protein kinase pathway," Journal of Immunology, vol. 182, no. 12, pp. 79978004, 2009.

[16] G. T. Brown and T. M. McIntyre, "Lipopolysaccharide signaling without a nucleus: kinase cascades stimulate platelet shedding of proinflammatory IL-1beta-rich microparticles," Journal of Immunology, vol. 186, no. 9, pp. 5489-5496, 2011.

[17] M. Czabanka, C. Peter, E. Martin, and A. Walther, "Microcirculatory endothelial dysfunction during endotoxemiainsights into pathophysiology, pathologic mechanisms and clinical relevance," Current Vascular Pharmacology, vol. 5, no. 4, pp. 266-275, 2007.

[18] D. Secor, F. Li, C. G. Ellis et al., "Impaired microvascular perfusion in sepsis requires activated coagulation and Pselectin-mediated platelet adhesion in capillaries," Intensive Care Medicine, vol. 36, no. 11, pp. 1928-1934, 2010.

[19] K. Tyml, "Critical role for oxidative stress, platelets, and coagulation in capillary blood flow impairment in sepsis," Microcirculation, vol. 18, no. 2, pp. 152-162, 2011.

[20] C. L. Bockmeyer, P. A. Reuken, T. P. Simon et al., "ADAMTS13 activity is decreased in a septic porcine model. Significance for glomerular thrombus deposition," Thrombosis and Haemostasis, vol. 105, no. 1, pp. 145-153, 2011.

[21] H. D. Flad and E. Brandt, "Platelet-derived chemokines: pathophysiology and therapeutic aspects," Cellular and Molecular Life Sciences, vol. 67, no. 14, pp. 2363-2386, 2010.

[22] A. S. Weyrich and G. A. Zimmerman, "Platelets: signaling cells in the immune continuum," Trends in Immunology, vol. 25, no. 9, pp. 489-495, 2004.

[23] S. R. Steinhubl, "Platelets as mediators of inflammation," Hematology/Oncology Clinics of North America, vol. 21, no. 1, pp. 115-121, 2007.

[24] A. R. L. Gear and D. Camerini, "Platelet chemokines and chemokine receptors: linking hemostasis, inflammation, and host defense," Microcirculation, vol. 10, no. 3-4, pp. 335-350, 2003.

[25] J. W. Semple and J. Freedman, "Platelets and innate immunity," Cellular and Molecular Life Sciences, vol. 67, no. 4, pp. 499-511, 2010.

[26] S. S. Smyth, R. P. Mcever, A. S. Weyrich et al., "Platelet functions beyond hemostasis," Journal of Thrombosis and Haemostasis, vol. 7, no. 11, pp. 1759-1766, 2009.

[27] E. I. B. Peerschke, W. Yin, and B. Ghebrehiwet, "Platelet mediated complement activation," Advances in Experimental Medicine and Biology, vol. 632, pp. 81-91, 2008.
[28] E. I. Peerschke, W. Yin, and B. Ghebrehiwet, "Complement activation on platelets: implications for vascular inflammation and thrombosis," Molecular Immunology, vol. 47, no. 13, pp. 2170-2175, 2010.

[29] J. Wiesner and A. Vilcinskas, "Antimicrobial peptides: the ancient arm of the human immune system," Virulence, vol. 1, no. 5, pp. 440-464, 2010.

[30] L. T. Nguyen, P. H. Kwakman, D. I. Chan et al., "Exploring platelet chemokine antimicrobial activity: nuclear magnetic resonance backbone dynamics of nap-2 and tc-1," Antimicrobial Agents and Chemotherapy, vol. 55, no. 5, pp. 2074-2083, 2011.

[31] S. R. Clark, A. C. Ma, S. A. Tavener et al., "Platelet TLR4 activates neutrophil extracellular traps to ensnare bacteria in septic blood," Nature Medicine, vol. 13, no. 4, pp. 463-469, 2007.

[32] F. Setzer, V. Oberle, M. Bläss et al., "Platelet-derived microvesicles induce differential gene expression in monocytic cells: a DNA microarray study," Platelets, vol. 17, no. 8, pp. 571-576, 2006.

[33] J. Winning, J. Neumann, M. Kohl et al., "Antiplatelet drugs and outcome in mixed admissions to an intensive care unit," Critical Care Medicine, vol. 38, no. 1, pp. 32-37, 2010.

[34] X. Bosch, J. Marrugat, and J. Sanchis, "Platelet glycoprotein IIb/IIIa blockers during percutaneous coronary intervention and as the initial medical treatment of non-ST segment elevation acute coronary syndromes," Cochrane Database of Systematic Reviews, no. 9, Article ID CD002130, 2010.

[35] M. W. Behan, D. P. Chew, and P. E. Aylward, "The role of antiplatelet therapy in the secondary prevention of coronary artery disease," Current Opinion in Cardiology, vol. 25, no. 4, pp. 321-328, 2010.

[36] T. S. Field and O. R. Benavente, "Current status of antiplatelet agents to prevent stroke," Current Neurology and Neuroscience Reports, vol. 11, no. 1, pp. 6-14, 2011.

[37] F. Catella-Lawson, M. P. Reilly, S. C. Kapoor et al., "Cyclooxygenase inhibitors and the antiplatelet effects of aspirin," The New England Journal of Medicine, vol. 345, no. 25, pp. 18091817, 2001.

[38] R. A. Chaer, J. A. Graham, and L. Mureebe, "Platelet function and pharmacologic inhibition," Vascular and Endovascular Surgery, vol. 40, no. 4, pp. 261-267, 2006.

[39] A. J. Ansara, S. A. Nisly, S. A. Arif, J. M. Koehler, and S. T. Nordmeyer, "Aspirin dosing for the prevention and treatment of ischemic stroke: an indication-specific review of the literature," Annals of Pharmacotherapy, vol. 44, no. 5, pp. 851-862, 2010.

[40] J. Herlitz, P. P. Tóth, and J. Næsdal, "Low-dose aspirin therapy for cardiovascular prevention: quantification and consequences of poor compliance or discontinuation," American Journal of Cardiovascular Drugs, vol. 10, no. 2, pp. 125-141, 2010.

[41] A. Lanas, "Gastrointestinal bleeding associated with low-dose aspirin use: relevance and management in clinical practice," Expert Opinion on Drug Safety, vol. 10, no. 1, pp. 45-54, 2011.

[42] S. Shakib, "Aspirin for primary prevention: do potential benefits outweigh the risks?" Internal Medicine Journal, vol. 39, no. 6, pp. 401-407, 2009.

[43] A. D. Michelson, "New P2Y12 antagonists," Current Opinion in Hematology, vol. 16, no. 5, pp. 371-377, 2009.

[44] M. Cattaneo, "The platelet P2Y receptor for adenosine diphosphate: congenital and drug-induced defects," Blood, vol. 117 , no. 7, pp. 2102-2112, 2011. 
[45] P. A. Gurbel, D. J. Kereiakes, and U. S. Tantry, "Ticagrelor for the treatment of arterial thrombosis," Expert Opinion on Pharmacotherapy, vol. 11, no. 13, pp. 2251-2259, 2010.

[46] S. A. Mousa, W. P. Jeske, and J. Fareed, "Prasugrel: a novel platelet ADP P2Y(12) receptor antagonist," Methods in Molecular Biology, vol. 663, pp. 221-228, 2010.

[47] A. W. J. Van't Hof and M. Valgimigli, "Defining the role of platelet glycoprotein receptor inhibitors in STEMI: focus on tirofiban," Drugs, vol. 69, no. 1, pp. 85-100, 2009.

[48] L. Bolognese, "The emerging role of platelet glycoprotein IIb/IIIa inhibitors in managing high-risk patients with nonST segment elevation acute coronary syndromes," Current Medical Research and Opinion, vol. 23, no. 6, pp. 1217-1226, 2007.

[49] H. Horiuchi, "Recent advance in antiplatelet therapy: the mechanisms, evidence and approach to the problems," Annals of Medicine, vol. 38, no. 3, pp. 162-172, 2006.

[50] P. M. Ridker, M. Cushman, M. J. Stampfer, R. P. Tracy, and C. H. Hennekens, "Inflammation, aspirin, and the risk of cardiovascular disease in apparently healthy men," The New England Journal of Medicine, vol. 336, no. 14, pp. 973-979, 1997.

[51] S. R. Steinhubl, J. J. Badimon, D. L. Bhatt, J. M. Herbert, and T. Lüscher, "Clinical evidence for anti-inflammatory effects of antiplatelet therapy in patients with atherothrombotic disease," Vascular Medicine, vol. 12, no. 2, pp. 113-122, 2007.

[52] J. Graff, S. Harder, O. Wahl, E. H. Scheuermann, and J. Gossmann, "Anti-inflammatory effects of clopidogrel intake in renal transplant patients: effects on platelet-leukocyte interactions, platelet CD40 ligand expression, and proinflammatory biomarkers," Clinical Pharmacology and Therapeutics, vol. 78, no. 5, pp. 468-476, 2005.

[53] J. B. Muhlestein, "Effect of antiplatelet therapy on inflammatory markers in atherothrombotic patients," Thrombosis and Haemostasis, vol. 103, no. 1, pp. 71-82, 2010.

[54] I. Ikonomidis, F. Andreotti, E. Economou, C. Stefanadis, P. Toutouzas, and P. Nihoyannopoulos, "Increased proinflammatory cytokines in patients with chronic stable angina and their reduction by aspirin," Circulation, vol. 100, no. 8, pp. 793-798, 1999.

[55] J. R. Fletcher, "Prostaglandin synthetase inhibitors in endotoxin or septic shock-a review," Advances in Shock Research, vol. 10, pp. 9-14, 1983.

[56] P. S. Rao, D. Cavanagh, and L. W. Gaston, "Endotoxic shock in the primate: effects of aspirin and dipyridamole administration," American Journal of Obstetrics and Gynecology, vol. 140, no. 8, pp. 914-922, 1981.

[57] U. Schaper, G. Lueddeckens, W. Forster, and D. W. Scheuch, "Inhibition of lipoxygenase (LOX) or of cyclooxygenase (COX) improves survival of rats in endotoxin shock," Biomedica Biochimica Acta, vol. 47, no. 10-11, pp. S282-S285, 1988.

[58] Y. Ohtaki, H. Shimauchi, T. Yokochi, H. Takada, and Y. Endo, "In vivo platelet response to lipopolysaccharide in mice: proposed method for evaluating new antiplatelet drugs," Thrombosis Research, vol. 108, no. 5-6, pp. 303-309, 2002.

[59] L. Zhao, Y. Ohtaki, K. Yamaguchi et al., "LPS-induced platelet response and rapid shock in mice: contribution of O-antigen region of LPS and involvement of the lectin pathway of the complement system," Blood, vol. 100, no. 9, pp. 3233-3239, 2002 .

[60] F. B. Taylor, B. S. Coller, A. C. K. Chang et al., "7e3 $\mathrm{f}\left(\mathrm{ab}^{\prime}\right) 2$, a monoclonal antibody to the platelet GPIIb/IIIa receptor, protects against microangiopathic hemolytic anemia and microvascular thrombotic renal failure in baboons treated with $\mathrm{C} 4 \mathrm{~b}$ binding protein and a sublethal infusion of Escherichia coli," Blood, vol. 89, no. 11, pp. 4078-4084, 1997.

[61] Q. Pu, E. Wiel, D. Corseaux et al., "Beneficial effect of glycoprotein IIb/IIIa inhibitor (AZ-1) on endothelium in Escherichia coli endotoxin-induced shock," Critical Care Medicine, vol. 29, no. 6, pp. 1181-1188, 2001.

[62] A. Walther, M. Czabanka, M. M. Gebhard, and E. Martin, "Glycoprotein IIB/IIIA-inhibition and microcirculatory alteraktions during experimental endotoxemia-an intravital microscopic study in the rat," Microcirculation, vol. 11, no. 1, pp. 79-88, 2004.

[63] V. Evangelista, S. Manarini, G. Dell'Elba et al., "Clopidogrel inhibits platelet-leukocyte adhesion and platelet-dependent leukocyte activation," Thrombosis and Haemostasis, vol. 94, no. 3, pp. 568-577, 2005.

[64] V. Evangelista, G. Dell'Elba, N. Martelli et al., "Anti-inflammatory effects of clopidogrel in the mouse," Journal of Thrombosis and Haemostasis, vol. 5, supplement 2, p. P-M-283, 2007.

[65] J. Winning, J. Reichel, Y. Eisenhut et al., "Anti-platelet drugs and outcome in severe infection: clinical impact and underlying mechanisms," Platelets, vol. 20, no. 1, pp. 50-57, 2009.

[66] M. Seidel, J. Winning, R. A. Claus, M. Bauer, and W. Lösche, "Beneficial effect of clopidogrel in a mouse model of polymicrobial sepsis," Journal of Thrombosis and Haemostasis, vol. 7, no. 6, pp. 1030-1031, 2009.

[67] S. Hagiwara, H. Iwasaka, A. Hasegawa et al., "Adenosine diphosphate receptor antagonist clopidogrel sulfate attenuates LPS-induced systemic inflammation in a rat model," Shock, vol. 35, no. 3, pp. 289-292, 2011.

[68] X. Tu, X. Chen, Y. Xie et al., "Anti-inflammatory renoprotective effect of clopidogrel and irbesartan in chronic renal injury," Journal of the American Society of Nephrology, vol. 19, no. 1, pp. 77-83, 2008.

[69] K. Schrör, "Aspirin and platelets: the antiplatelet action of aspirin and its role in thrombosis treatment and prophylaxis," Seminars in Thrombosis and Hemostasis, vol. 23, no. 4, pp. 349356, 1997.

[70] R. F. Storey, "Biology and pharmacology of the platelet P2Y12 receptor," Current Pharmaceutical Design, vol. 12, no. 10, pp. 1255-1259, 2006.

[71] S. Christensen, R. W. Thomsen, M. B. Johansen et al., "Preadmission statin use and one-year mortality among patients in intensive care-a cohort study," Critical Care, vol. 14, no. 2, p. R29, 2010.

[72] I. Kouroumichakis, N. Papanas, S. Proikaki, P. Zarogoulidis, and E. Maltezos, "Statins in prevention and treatment of severe sepsis and septic shock," European Journal of Internal Medicine, vol. 22, no. 2, pp. 125-133, 2011.

[73] H. R. O’Neal Jr., T. Koyama, E. A. Koehler et al., "Prehospital statin and aspirin use and the prevalence of severe sepsis and acute lung injury/acute respiratory distress syndrome," Critical Care Medicine, vol. 39, no. 6, pp. 1343-1359, 2011.

[74] S. Janda, A. Young, J. M. FitzGerald, M. Etminan, and J. Swiston, "The effect of statins on mortality from severe infections and sepsis: a systematic review and meta-analysis," Journal of Critical Care, vol. 25, no. 4, pp. 656.e7-656.e22, 2010.

[75] G. C. Makris, G. Geroulakos, M. C. Makris, D. P. Mikhailidis, and M. E. Falagas, "The pleiotropic effects of statins and omega-3 fatty acids against sepsis: a new perspective," Expert Opinion on Investigational Drugs, vol. 19, no. 7, pp. 809-814, 2010 
[76] D. Viasus, C. Garcia-Vidal, F. Gudiol, and J. Carratalà, "Statins for community-acquired pneumonia: current state of the science," European Journal of Clinical Microbiology and Infectious Diseases, vol. 29, no. 2, pp. 143-152, 2010.

[77] J. M. Erlich, D. S. Talmor, R. Cartin-Ceba, and D. J. Kor, "Prehospitalization antiplatelet therapy is associated with a reduced incidence of acute lung injury: a population-based cohort study," Chest, vol. 139, no. 2, pp. 289-295, 2011.

[78] D. J. Kor, J. Erlich, M. N. Gong et al., "Association of prehospitalization aspirin therapy and acute lung injury: Results of a multicenter international observational study of at-risk patients," Critical Care Medicine, vol. 39, no. 22, pp. 23932400, 2011.

[79] G. J. Blake and P. M. Ridker, "Inflammatory bio-markers and cardiovascular risk prediction," Journal of Internal Medicine, vol. 252, no. 4, pp. 283-294, 2002.

[80] S. Steffens and F. Mach, "Inflammation and atherosclerosis," Herz, vol. 29, no. 8, pp. 741-748, 2004.

[81] H. Loppnow, K. Werdan, and M. Buerke, "Vascular cells contribute to atherosclerosis by cytokine- and innate-immunityrelated inflammatory mechanisms," Innate Immunity, vol. 14, no. 2, pp. 63-87, 2008.

[82] C. I. Vardavas and D. B. Panagiotakos, "The causal relationship between passive smoking and inflammation on the development of cardiovascular disease: a review of the evidence," Inflammation and Allergy - Drug Targets, vol. 8, no. 5, pp. 328333, 2009.

[83] P. G. Chassot, A. Delabays, and D. R. Spahn, "Perioperative use of anti-platelet drugs," Best Practice and Research, vol. 21, no. 2, pp. 241-256, 2007.

[84] E. Hijazi, "Aspirin does dot increase bleeding and allogeneic blood transfusion in coronary artery surgery," Thoracic and Cardiovascular Surgery, vol. 59, no. 7, pp. 421-424, 2011.

[85] G. Kotsovolis, G. Komninos, A. Kyrgidid, and D. Papadimitriou, "Preoperative withdrawal of antiplatelet treatment in lower limb vascular patients prior to surgical management under epidural or spinal anaesthesia: an evidence based approach and systematic review," International Angiology, vol. 29, no. 6, pp. 475-481, 2010.

[86] P. F. Dineen, R. J. Curtin, and J. A. Harty, "A review of the use of common antiplatelet agents in orthopaedic practice," Journal of Bone and Joint Surgery B, vol. 92, no. 9, pp. 11861191, 2010. 


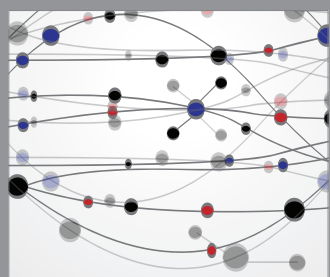

The Scientific World Journal
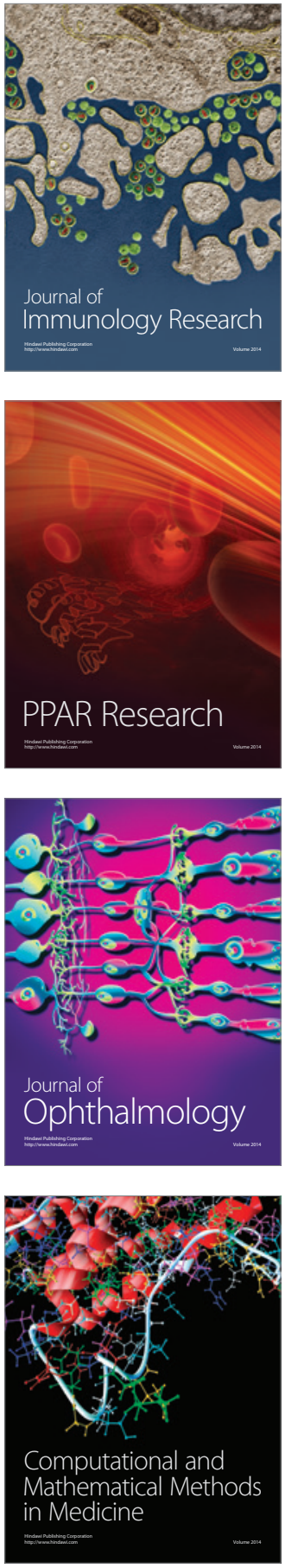

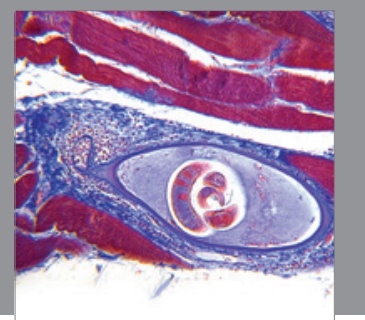

Gastroenterology

Research and Practice
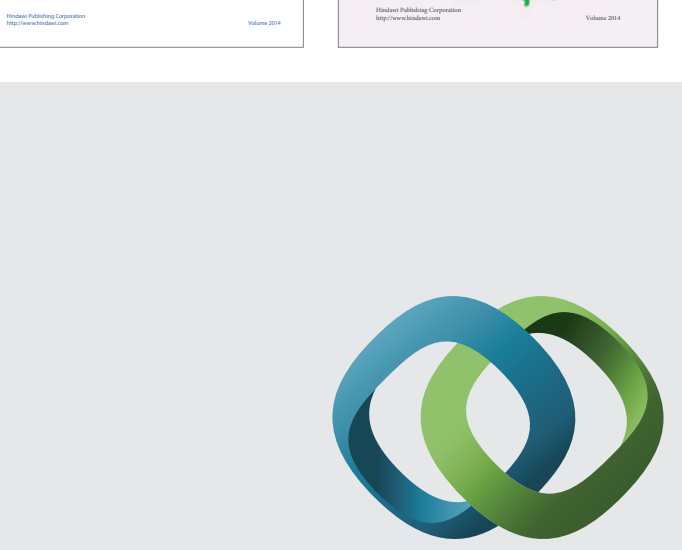

\section{Hindawi}

Submit your manuscripts at

http://www.hindawi.com
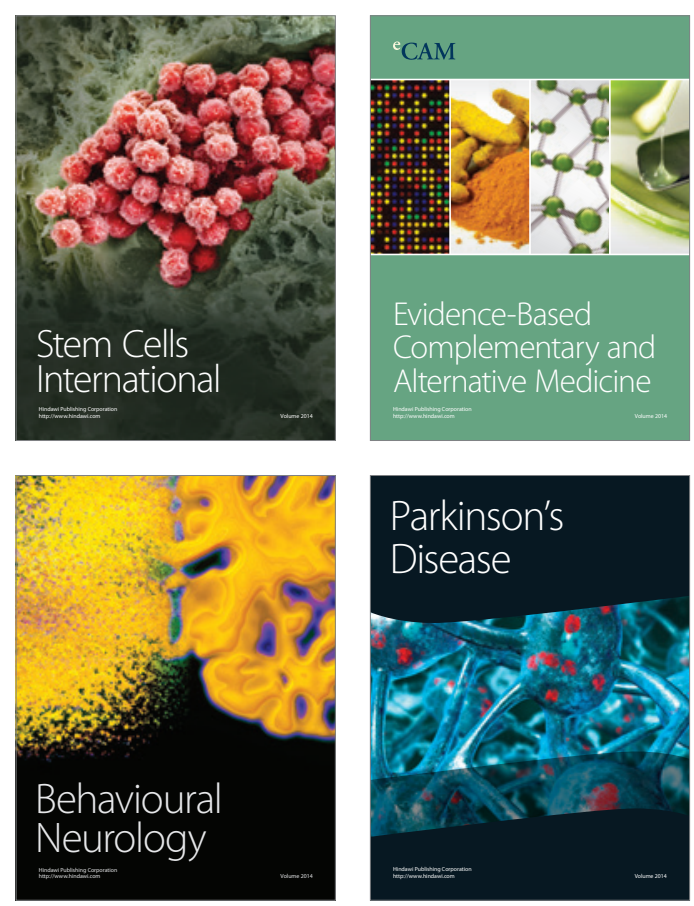

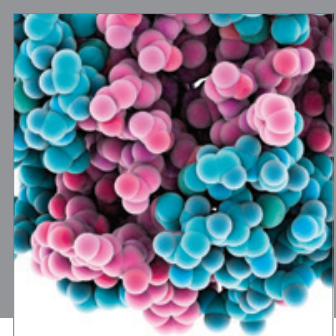

Journal of
Diabetes Research

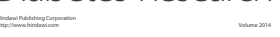

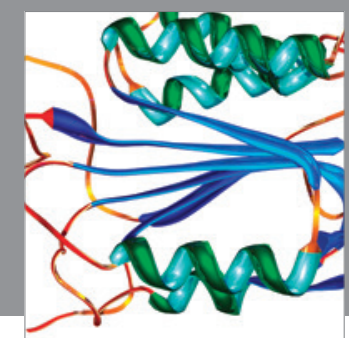

Disease Markers
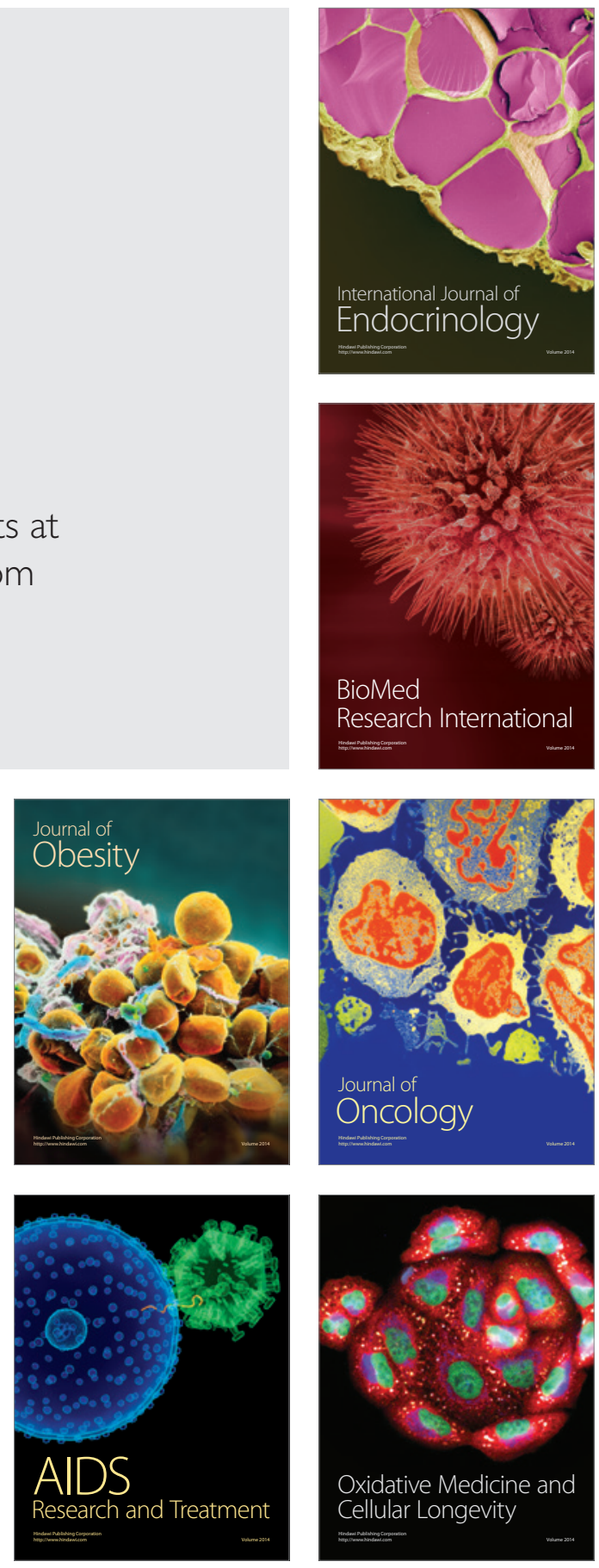